\title{
Management of a Rare Case of Post IVF Triplet Ectopic Pregnancy Post Bilateral Tubal Occlusion
}

\author{
Abha Majumdar and Poonam Mishra* \\ Fellow in Reproductive Medicine, Sir Ganga Ram Hospital, India
}

*Corresponding author: Poonam Mishra, Fellow in Reproductive Medicine, Centre of IVF and Human Reproduction, Sir Ganga Ram Hospital, New Delhi, India

Submission: 啙 December 12, 2017; Published: 眥 September 10, 2018

\begin{abstract}
Incidence of ectopic pregnancy after Invitro fertilization and embryo transfer (IVF-ET) in patients with prior bilateral tubal occlusion is negligible and if it occurs, it happens at unusual sites which are both difficult to diagnose as well as to treat. The possibility of early uterine rupture with life threatening haemorrhage is very high in such cases, therefore treatment of these pregnancies often require hysterectomy as a life saving measure. Our case of triplet lives ectopic pregnancy followed embryo transfer of three blastocysts. She had undergone laparoscopic bilateral proximal tubal occlusion 5 years ago. In this case, trans-abdominal ultrasound guided suction evacuation was attempted unsuccessfully. Hysteroscopy followed, which confirmed normal endometrial cavity with no gestational sac within it. Post adhesiolysis and bowel dissection, left sided cornual bulge was seen suggestive of left isthmic pregnancy. Putrescin was injected, incision made, products evacuated, and hemostatic sutures applied. On the right side, post bowel dissection hydrosalpinx seen and drained followed by putrescin injection at uterine angle. While de-roofing hydrosalpinx from the angle of uterus, products of conception were seen to extrude from posterior side of the uterine musculature at the angular area, which were evacuated, and hemostatic sutures applied. This case highlights the importance as well as need of using a gambit of diagnostic and operative procedures to enable treatment of such complicated cases successfully. This case report also highlights the misery and complications which could follow transfer of multiple good quality blastocysts especially if these implant at ectopic sites.
\end{abstract}

Keywords: Invitro fertilisation (IVF); Ectopic pregnancy; Tubal occlusion; IVF-ET: Invitro fertilization and embryo transfer

Abbreviations: IVF: Invitro Fertilisation; $\beta$ hCG: Human Chorionic Gonadotropin; ERA: Endometrial Receptivity Assay

\section{Introduction}

Incidence of ectopic pregnancy after Invitro fertilization and embryo transfer (IVF-ET) in patients with prior bilateral tubal occlusion is negligible, yet it can occur at the isthmic stump, cornual or angle of uterus. The possibility of early uterine rupture with

life threatening haemorrhage is very high in such cases, therefore treatment of these pregnancies often require hysterectomy as a life saving measure. The difficulties in management of such ectopic pregnancies especially if one needs to save the uterus is immense, more so in high order ectopic pregnancies.

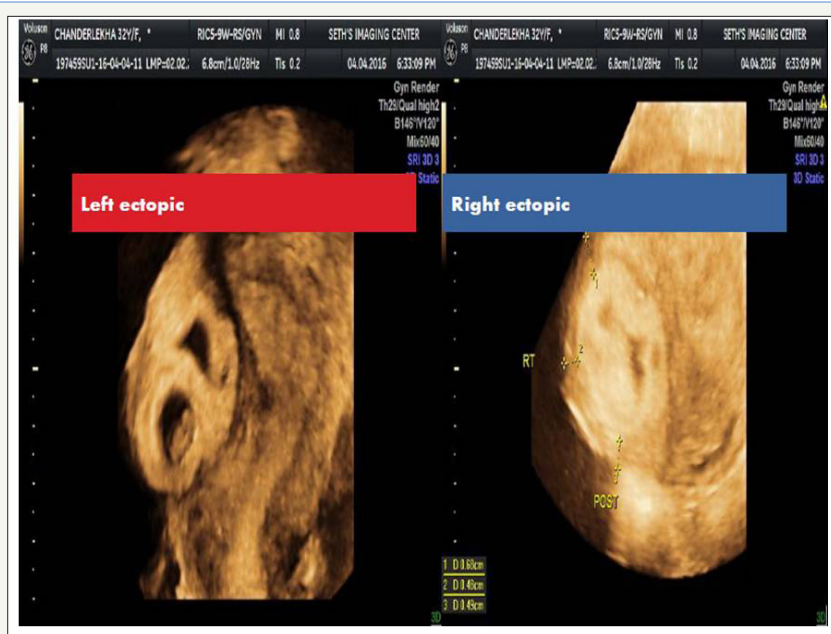

Figure 1: Image of transvaginal ultrasound showing ectopic mass in isthmic region on left site and on right side ectopic mass at angle of uterus. 
We hereby report a case of triplet live ectopic pregnancy of 6 weeks 5 days gestational age with beta human chorionic gonadotropin ( $\beta$ hCG) more than 200000 units (2sacs in right cornual and $1 \mathrm{in} \mathrm{left} \mathrm{isthmus)} \mathrm{following} \mathrm{embryo} \mathrm{transfer} \mathrm{of} \mathrm{three}$ blastocysts (Figure 1). This patient had undergone laparoscopic bilateral proximal tubal occlusion 5 years ago for bilateral hydrosalpinx with frozen pelvis. She also had history of 6 failed IVF-ET cycles at different centers for tubal factor infertility before she was referred to us, for management of this ectopic pregnancy.

\section{Management}

Patient was put up for surgery after all necessary pre-operative details were taken care off. It has been seen that angular pregnancies may evade diagnosis by real time ultrasonography and sometimes also with 3D ultrasounds [1]. In some cases the possibility of an intrauterine pregnancy very close to the angle cannot be distinguished from true angular pregnancies. Considering these facts, in this case as the first step, trans-abdominal ultrasound guided suction evacuation was attempted unsuccessfully. Hysteroscopy followed, which confirmed normal endometrial cavity with no gestational sac within it hence the diagnosis of angular/cornual pregnancy was confirmed. Laparoscopy was attempted but converted to laparotomy due to very little visibility structures due to frozen pelvis. Post adhesiolysis and bowel dissection, bulky uterus with left sided cornual bulge was seen suggestive of left isthmic pregnancy. Putrescin was injected, incision made, products evacuated, and hemostatic sutures applied. On the right side, post bowel dissection hydrosalpinx seen and drained followed by putrescin injection at uterine angle. While de-roofing hydrosalpinx from the angle of uterus, products of conception were seen to extrude from posterior side of the uterine musculature at the angular area, which were evacuated, and hemostatic sutures applied. Intra operative ultrasound was used to confirm complete clearance of both sacs before closing the abdomen. Post-operative period was uneventful.

\section{Discussion}

This case has been presented to high light two issues pertinent to the reproductive physician, apart from bringing forward the role of using multiple intra operative diagnostic modalities in management of such complicated cases. Firstly, the primary aim of us physicians while treating any patient continues to be 'Primum Nor Nocera' which means 'cause no harm' and the responsibility is greater to us as reproductive physicians where we treat young healthy women seeking help to make or complete their families. There is a trend towards multiple embryo transfer for women with repeated IVF failures. However, one needs to counterbalance the desire for a pregnancy vis-a-vis the risk of multiple pregnancies which can become a nightmare in case it happens at rare ectopic sites especially in a woman with frozen pelvis with clipped tubes.

Theoretically, ectopic pregnancy after IVF-ET should be a rare phenomenon as embryos are transferred directly inside the uterine cavity. However, the world's first reported in-vitro fertilization and embryo transfer pregnancy was a tubal ectopic [2]. This should be even rarer in women whose tubes have been clipped. Nevertheless, in less than $1 \%$ of women these embryos can still implant in ectopic sites such as angle of the uterus, interstitial area (also known as cornual), isthmic stump as well as into the cervix and rarely in the abdominal cavity [3]. Concerns have already been raised regarding unselective use of preventive salpingectomy before IVF [4]. Earlier such ectopic and heterotopic conceptions, which were considered very rare types of pregnancies, are now being seen more often than before, and are attributed to conceptions arising as consequence of post salpingectomy IVF-ET. Occurrence of interstitial pregnancy is estimated to be as low as 1:3600 for all pregnancies achieved with IVF treatments but bilateral salpingectomy markedly increases the risk for interstitial ectopic with their antecedent risk of uterine rupture [5,6]. The risk of rupture uterus with life threatening haemorrhage often necessitates the need of hysterectomy in these women [7]. For infertile couples' difficulties in management of such ectopic pregnancies is even greater where there is need to save the uterus.

The second issue which comes forward from this case is the possibility of altered implantation potential of the uterine endometrium in these women. In this case six attempts of IVF-ET failed and eventually in the seventh cycle after transferring 3 good quality blastocysts into the uterus, all of them choose an ectopic site rather than the uterine endometrium to implant. It appears that uterine receptivity may be altered in some women where the implantation window may be different from the time appropriate for the embryos to hatch and implant. The window of implantation of the tubal endothelium may precede or follow a closed window of implantation of uterine endometrium. The role of endometrial receptivity assay (ERA) in recurrent implantation failure has been explored in IVF cycles where 2 or more high quality embryos have failed to implant in the uterine endometrium [8,9]. However, it is in cases where embryos placed in the uterus fail to implant on the uterine endometrium, but roll on to implant in an ectopic site, raises questions towards the role of ERA in such women too.

\section{Conclusion}

This case highlights the importance as well as need of using a gambit of diagnostic and operative procedures in absolute synchrony to enable treatment of such complicated cases successfully. This case report also highlights the misery and complications which could follow transfer of multiple good quality blastocysts especially if these implant at ectopic sites.

\section{References}

1. Arleo EK, DeFilippis EM (2014) Cornual, interstitial, and angular pregnancies: Clarifying the terms and a review of the literature. Clin Imaging 38(6): 763-770.

2. Steptoe PC, Edwards RG (1976) Reimplantation of a human embryo with subsequent tubal pregnancy. Lancet 1(7965): 880-882.

3. Wang J, Huang D, Lin X, Saravelos SH, Chen J, et al. (2016) Incidence of interstitial pregnancy after in vitro fertilization/embryo transfer and the outcome of a consecutive series of 38 cases managed by laparoscopic cornuostomy or cornual repair. J Minim Invasive Gynecol 23(5): 739747.

4. Puttemans P, Brosens I (1996) Salpingectomy improves in-vitro fertilization outcome in patients with hydrosalpinx: blind victimization of the fallopian tube? Hum Reprod 11(10): 2079-2081. 
5. Garavaglia E, Quaranta L, Redaelli A, Colombo G, Pasi F, et al. (2012) Interstitial pregnancy after in vitro fertilization and embryo transfer following bilateral salpingectomy: report of two cases and literature review. Int J Fertil Steril 6(2): 131-134

6. Agarwal SK, Wisot AL, Garzo G, Meldrum DR (1996) Cornual pregnancies in patients with prior salpingectomy undergoing in vitro fertilization and embryo transfer. Fertil Steril 65(3): 659-660.

7. Moawad NS, Mahajan ST, Moniz MH, Taylor SE, Hurd WW (2010) Current diagnosis and treatment of interstitial pregnancy. Am J Obstet Gynecol 202(1): 15-29.
8. Ruiz Alonso M, Blesa D, Díaz Gimeno P, Gómez E, Fernández Sánchez $M$, et al. (2013) The endometrial receptivity array for diagnosis and personalized embryo transfer as a treatment for patients with repeated implantation failure. Fertil Steril 100(3): 818-824.

9. Mahajan N (2015) Endometrial receptivity array: Clinical application. J Hum Reprod Sci 8(3): 121-129.
Creative Commons Attribution 4.0 International License

For possible submissions Click Here

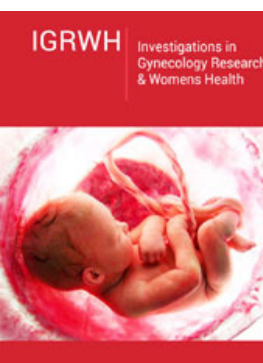

Investigations in Gynecology Research \& Womens Health

\section{Benefits of Publishing with us}

- High-level peer review and editorial services

- Freely accessible online immediately upon publication

- Authors retain the copyright to their work

- Licensing it under a Creative Commons license

- Visibility through different online platforms 